\title{
Eficacia del ácido hialurónico en el tratamiento de los trastornos temporomandibulares. Revisión sistemática
}

\section{Effectiveness of hialuronic acid in temporomandibular disorders treatment. A systematic review}

J. Jara Armijos*, B. Hidalgo Andrade**, B. Velásquez Ron***

\section{RESUMEN}

Los trastornos temporomandibulares constituyen una entidad clínica que involucra la articulación temporomandibular, los músculos masticatorios y estructuras circundantes. Entre las opciones de tratamiento, el ácido hialurónico $(\mathrm{AH})$ se ha utilizado en variadas concentraciones, peso molecular y protocolos de inyección, lo que podría variar el perfil de eficacia en los resultados del tratamiento de TTM.

Objetivo: Descubrir mediante una revisión sistemática el mejor protocolo de aplicación de AH para el tratamiento de TTM.

Método: Se realizó una búsqueda en Pubmed, Medline, Scopus, Cochrane, Dentistry \& Oral Science Source, Complementary Index y Academic search complete, con las palabras: "ácido hialurónico"; "Artrocentesis ácido hialurónico"; "trastornos temporomandibulares" considerando ensayos clínicos aleatorizados en humanos hasta con diez años de antigüedad, se preseleccionaron 71 artículos y a través del diagrama de flujo prisma se seleccionaron 22 artículos evaluados en la escala Jadad.

Resultados: Las modalidades de tratamiento incluyeron: AH versus AINES, betametasona, tenoxicam o glucosamida oral (4 estudios); artrocentesis más AH versus artrocentesis con plasma rico en plaquetas ( 2 estudios) y plasma rico en factor de crecimiento (1 estudio); artrocentesis más AH versus artrocentesis con solución salina o lactato de ringer (4 estudios); artrocentesis más AH versus artrocentesis sola (3 estudios); aplicaciones cíclicas de AH (6 estudios); y ciclos de inyecciones de AH versus terapia de férula oclusal (2 estudios). El período de seguimiento para la mayoría de estudios fue hasta de 12 meses.

Conclusión: Los protocolos basados en aplicaciones cíclicas de inyecciones de $\mathrm{AH}$, demostraron mejores resultados en el alivio de los síntomas de TTM a largo plazo.

PALABRAS CLAVE: Ácido hialurónico; Artrocentesis ácido hialurónico; Trastornos temporomandibulares.

\section{ABSTRACT}

Temporomandibular disorders (TMD) embraces a number of clinical problems involving the masticatory muscles, the temporomandibular joint (TMJ) and associated structures. TMD treatment options include hyaluronic acid (HA) injections that have been used with different concentrations, molecular weight and

* Odontólogo General. Universidad de Loja Estudiante de Postgrado de Rehabilitación Oral.

Universidad de las Américas ORCID ID: https://orcid.org/0000-0002-2395-1349.

* * Odontóloga General. Universidad Estatal de Guayaquil Estudiante de Postgrado de Rehabilitación Oral. Universidad de las Américas

*** Phd Universidad de New York Universidad de las Américas Afiliación: Departamento de prótesis Facultad de Odontología Universidad de las Américas ORCID ID https://orcid.org/0000-0001-5660-3941. 
injection protocol, which could vary the efficacy profile in the TMD treatment results.

Objective: Discover through a systematic review the best HA application protocol for the treatment of TMD. Method: A search was performed in Pubmed, Medline, Scopus, Cochrane, Dentistry E Oral Science Source, Complementary Index and Academic search complete, The search included terms such as "hyaluronic acid", "temporomandibular disorders", and "arthrocentesis with hyaluronic acid" in randomized clinical trials with human subjects from up to ten years ago. Of the 71 articles screened, 22 articles were included in the PRISMA flow-diagram and were assessed with the Jadad scale.

Results: Treatment modalities consisted of the following: four studies in HA versus other types of medications such as non-steroidal anti-inflammatory drugs (NSAIDs), betamethasone, tenoxicam or oral glucosamine; two studies in arthrocentesis plus HA versus arthrocentesis with platelet rich plasma (PRP); and one study in plasma rich in growth factor (PRFC); four studies in arthrocentesis plus HA versus arthrocentesis with saline or ringer's lactate solution; three studies in arthrocentesis plus HA versus arthrocentesis alone; six studies in cyclic applications of HA; and two studies in cycles of HA injections versus occlusal splint therapy. The follow-up period for most studies was up to 12 months.

Conclusion: The protocols based on cyclic applications of injections of HA, demonstrated better results in the relief of long-term symptoms of TMD.

KEY WORDS: Hyaluronic acid; Hyaluronic acid arthrocentesis; Temporomandibular disorders.

Fecha de recepción: 7 de octubre de 2019

Fecha de aceptación: 10 de noviembre de 2019

J. Jara, B. Hidalgo, B. Velásquez. Eficacia del ácido hialurónico en el tratamiento de los trastornos temporomandibulares. Revisión sistemática. Av. Odontoestomatol. 2020; 36, (1): 23-47.

\section{INTRODUCCIÓN}

Los trastornos temporomandibulares (TTM) constituyen una entidad clínica que involucra la articulación temporomandibular, músculos masticatorios y estructuras circundantes. Se considera como una condición patológica muy común que afecta entre el $5 \%$ y $12 \%$ de la población general; posiblemente influenciada por múltiples factores etiológicos y pato-fisiológicos dentro de los que se incluyen principalmente factores mecánicos como la sobrecarga articular o bruxismo y los procesos inflamatorios propios que, combinados, pueden determinar la degeneración gradual de los elementos que componen la articulación: disco, cartílago, cóndilo mandibular, membrana y líquido sinovial. ${ }^{(1)}$

El desajuste interno de la articulación temporomandibular (ATM), que representa el $80 \%$ a $90 \%$ de los casos, se define como una relación anormal entre el disco interarticularlar y el cóndilo, a menudo asociado a cambios degenerativos u osteoartritis del cóndilo. Clínicamente, se caracterizan por dolor articular, apertura bucal limitada y ruidos articulares que producen una disminución en la calidad de vida de los pacientes. ${ }^{(2)}$
Como opciones de tratamiento de los TTM, el no quirúrgico es efectivo en la mayoría de los casos reduciendo el dolor y mejorando la movilidad de la mandíbula; dejando procedimientos mínimamente invasivos como artrocentesis, lisis y lavado artroscópicos, o artroscopia quirúrgica, aproximadamente el $10 \%$ de los casos refractarios al tratamiento conservador. ${ }^{(2-3)}$.

El ácido hialurónico (AH) es un glicosaminoglicano de alto peso molecular cuyo reservorio más grande en el organismo es el líquido sinovial de las articulaciones diartrodias, donde las moléculas de $\mathrm{AH}$ se sintetizan principalmente por los sinoviocitos de tipo $B$, liberando una población de AH polidispersada con peso molecular (Pm) en el rango entre 2x106 y 10x106 Da y concentraciones de 0.5-4 mg/ml. La alta concentración de HA en líquido sinovial es esencial para el funcionamiento normal de la articulación, ya que le confiere propiedades excepcionales de viscoelasticidad y lubricación, responsables de la absorción de impactos en condiciones de alta compresión o fuerza, y lubricación en estados de reposo. Estas propiedades reológicas únicas del $\mathrm{AH}$ no solo reducen el desgaste del cartílago articular durante el movimiento de la articulación, sino que también 
estabilizan las articulaciones a bajas tasas de cizallamiento.

Inyecciones de AH en la ATM se han utilizado durante años como procedimientos únicos o asociadas con otros procedimientos (artrocentesis o la artroscopia) con buenos resultados. ${ }^{(4)}$ Dentro de los efectos del AH exógeno están el aumento de la elasticidad, la viscosidad del líquido sinovial, especialmente en las articulaciones en proceso degenerativo. Propiedades reparadoras a través de la formación de complejos con fosfolípidos en la membrana sinovial e incluso propiedades analgésicas. ${ }^{(5)}$

Las preparaciones de AH para administración intraarticular varían significativamente en concentración, peso molecular y protocolo de inyección ${ }^{(6)}$, incluso pueden diferir en términos de organización molecular así como en los materiales derivados químicamente que se diseñan para aumentar la elastoviscosidad y el tiempo de residencia intraarticular; por lo tanto, las posibles diferencias en el perfil de eficacia de cada producto pueden afectar los resultados en el tratamiento de los TTM, razón por la cual se plantea la presente revisión sistemática con el fin de evaluar la eficacia del ácido hialurónico en TTM bajo distintos protocolos.

\section{FIGURA1. FLUJOGRAMA DE SELECCIÓN DE ESTUDIOS}

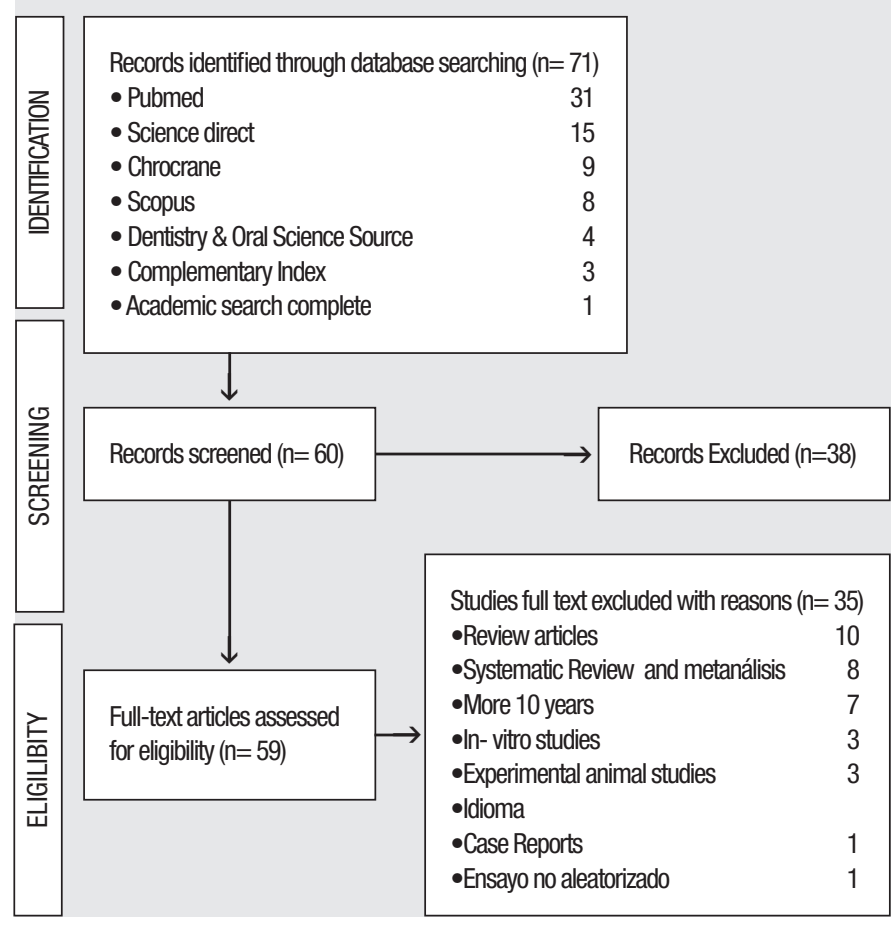

\section{MATERIAL Y MÉTODOS}

Para la búsqueda inicial se realiza se realiza un flujograma prisma y se seleccionan 71 artículos (ensayos clínicos aleatorizados) con restricción de lenguaje, utilizando la palabra clave "ácido hialurónico" "trastornos temporomandibulares" y "hyaluronic acid", "temporomandibular disorders" en diferentes fuentes de información y búsqueda de Pubmed, Science direct, Chrocrane, y Scopus.(Figura 1).

\section{Criterios de Selección}

Los artículos fueron seleccionados de acuerdo a los siguientes criterios de inclusión:

- Ensayos clínicos en seres humanos donde se estudia eficacia del ácido hialurónico en el tratamiento de los trastornos temporomandibulares

- Texto completo

-Artículos de más 10 años de vigencia

Los criterios de exclusion fueron:

-Ensayos clínicos en animales

- Revisiones sistemáticas

-Estudios in vitro

- Reportes de casos

- Revisiones de literatura

-Artículos de más de 10 años de vigencia

\section{RESULTADOS}

En la búsqueda inicial se encontró un total de 71 artículos. Después de la exclusión de duplicados, quedaron 61 artículos; seleccionados después de la evaluación cuidadosa de sus títulos y resúmenes. Luego de acceder a los textos completos de estos artículos finalmente se eligió 22 artículos que cumplieron con todos los criterios de inclusión. Todos los artículos se valoraron con la escala Jadad y se obtuvo: siete estudios con una puntuación de 5, cuatro obtuvieron una puntuación de 4 y siete obtuvieron una puntuación de 3, para un total de dieciocho estudios de alta calidad; y solo cuatro estudios de baja calidad. (Tabla 1 ).

\section{Características del estudio}

Las principales características de los estudios incluidos se detallan en la Tabla 2. Veinte y dos artículos fueron Ensayos clínicos y catorce fueron Ensayos 


\begin{tabular}{|c|c|c|c|c|c|c|}
\hline ESTUDIO & $\begin{array}{l}\text { Estudio descrito } \\
\text { como aleatorizado }\end{array}$ & $\begin{array}{l}\text { Aleatorización apropiada y } \\
\text { bien descrita en el artículo }\end{array}$ & $\begin{array}{l}\text { Estudio descrito } \\
\text { como doble ciego }\end{array}$ & $\begin{array}{l}\text { Método doble } \\
\text { ciego apropiado }\end{array}$ & $\begin{array}{l}\text { Descripción de } \\
\text { errores/deserción }\end{array}$ & TOTAL \\
\hline Femandez Ferrero M, et al. 2017 (22) & $\mathrm{Sl}$ & $\mathrm{Sl}$ & NO & NO & $\mathrm{SI}$ & 3 \\
\hline Su N, etal. $2014(23)$ & NO & NO & NO & NO & $\mathrm{Sl}$ & 1 \\
\hline Xao C, etal. 2018 (24) & $\mathrm{SI}$ & $\mathrm{SI}$ & $\mathrm{SI}$ & $\mathrm{Sl}$ & $\mathrm{Sl}$ & 5 \\
\hline KorkmazYT, etal. 2016 (25) & $\mathrm{Sl}$ & $\mathrm{Sl}$ & $\mathrm{Sl}$ & NO & $\mathrm{Sl}$ & 4 \\
\hline Comertk, etal. $2016(7)$ & $\mathrm{SI}$ & $\mathrm{Sl}$ & SI & NO & $\mathrm{SI}$ & 4 \\
\hline Gencer ZK, et al. 2014 (8) & $\mathrm{Sl}$ & $\mathrm{Sl}$ & $\mathrm{Sl}$ & $\mathrm{Sl}$ & $\mathrm{Sl}$ & 5 \\
\hline Manfredini D, et al. 2013. (13) & NO & NO & SI & NO & NO & 2 \\
\hline Triantaffilidou K, et al.2013 (14) & NO & NO & $\mathrm{Sl}$ & $\mathrm{Sl}$ & $\mathrm{SI}$ & 3 \\
\hline Guarda-Nardini L, etal. 2011 (9) & NO & NO & SI & $\mathrm{SI}$ & $\mathrm{Sl}$ & 3 \\
\hline Morey-Mas M, eta al. 2010 (17) & $\mathrm{SI}$ & $\mathrm{Sl}$ & $\mathrm{Sl}$ & $\mathrm{Sl}$ & $\mathrm{Sl}$ & 5 \\
\hline Manfredini D,et al 2009. (10) & NO & NO & SI & $\mathrm{SI}$ & $\mathrm{Sl}$ & 3 \\
\hline Tuncel U. 2012 (12) & NO & NO & $\mathrm{Sl}$ & $\mathrm{SI}$ & $\mathrm{Sl}$ & 3 \\
\hline Guarda-Nardini L, etal. 2012 (11) & $\mathrm{SI}$ & $\mathrm{SI}$ & SI & NO & $\mathrm{SI}$ & 4 \\
\hline Ozdamar S, etal. 2016 (16) & $\mathrm{Sl}$ & $\mathrm{Sl}$ & Sl & $\mathrm{SI}$ & $\mathrm{Sl}$ & 5 \\
\hline Patel P, et al. 2016 (15 & $\mathrm{Sl}$ & $\mathrm{SI}$ & $\mathrm{SI}$ & $\mathrm{SI}$ & NO & 4 \\
\hline Li C, etal. 2014 (20) & $\mathrm{Sl}$ & $\mathrm{Sl}$ & $\mathrm{Sl}$ & $\mathrm{Sl}$ & $\mathrm{SI}$ & 5 \\
\hline Ungor C, etal. 2015 (27) & NO & NO & NO & NO & $\mathrm{Sl}$ & 1 \\
\hline Bergstrand S. et al. 2019 (18) & $\mathrm{Sl}$ & $\mathrm{SI}$ & $\mathrm{SI}$ & $\mathrm{Sl}$ & $\mathrm{Sl}$ & 5 \\
\hline Tolga K, eta al. 2016 (21) & $\mathrm{Sl}$ & $\mathrm{Sl}$ & $\mathrm{SI}$ & $\mathrm{SI}$ & $\mathrm{SI}$ & 5 \\
\hline Małgorzata P, etal. 2017 (4) & NO & $\mathrm{Sl}$ & NO & NO & NO & 1 \\
\hline Gurung T, etal. 2017 (8) & $\mathrm{SI}$ & $\mathrm{Sl}$ & NO & NO & $\mathrm{SI}$ & 3 \\
\hline Tang Y, et al.2010 (19) & $\mathrm{Sl}$ & $\mathrm{Sl}$ & NO & NO & $\mathrm{Sl}$ & 3 \\
\hline
\end{tabular}

Clínicos Aleatorizados (ECAs). La principal patología encontrada fue osteartritis de ATM, el índice de valoración más utilizado fue le RDC/TMD (Axis I). La principal terapia indicada para la patología articular fue artrocentesis. Las modalidades de tratamiento incluyeron: AH versus otros tipos de medicamentos como AINES, betametasona, tenoxicam o glucosamida oral (4 estudios); artrocentesis más $\mathrm{AH}$ versus artrocentesis con plasma rico en plaquetas PRP (2 estudios) y plasma rico en factor de crecimiento PRFC (1 estudio); artrocentesis más $\mathrm{AH}$ versus artrocentesis con solución salina o lactato de Ringer (4 estudios); artrocentesis más AH versus artrocentesis sola (3 estudios); aplicaciones cíclicas de $\mathrm{AH}$ (6 estudios); y ciclos de inyecciones de $\mathrm{AH}$ versus terapia de férula oclusal (2 estudios). El período de seguimiento para la mayoría de los estudios 12 meses.

No se reportó el peso molecular del AH utilizado (16 estudios); en 3 estudios se utilizó $\mathrm{AH}$ de alto peso molecular y en 3 de bajo peso molecular.

En general todos los pacientes que recibieron inyecciones intraarticulares de $\mathrm{AH}$ mostraron mejores resultados en alivio del dolor y/o función articular en el posoperatorio inmediato que se mantuvieron hasta 6 meses después en comparación a los otros grupos analizados, especialmente en inyecciones cíclicas. De todas las terapias utilizadas sólo el PRFC mostró mejore resultados a largo plazo frente al $\mathrm{AH}$.
Aunque se incluyeron ECAs en esta revisión, no se realizó un metanálisis debido a la amplia diversidad clínica entre los estudios.

\section{DISCUSIÓN}

Varios tratamientos se han propuesto para el tratamiento de los trastornos temporomandibulares (TTM), conservadores (fisioterapia y férulas oclusales) hasta quirúrgicos como reemplazo articulares; la evidencia científica sugiere que el daño en la lubricación articular es el principal factor en la patogénesis de los cambios inflamatorios degenerativos, llevando a la introducción de la viscosuplementación y las inyecciones de ácido hialurónico $(\mathrm{AH})$ como un tratamiento prometedor para estos trastornos. Hallazgos preliminares alentadores demostraron la eficacia de las inyecciones de $\mathrm{AH}$ solas o en combinación con artrocentesis para el alivio del dolor, mejorando síntomas de TTM, no hay un protocolo establecido como "gold estándar" para el manejo de esta patología, objetivo de esta revisión sistemática.

De acuerdo con la escala Jadad, la mayoría de los ensayos clínicos incluidos en el estudio (90\%) demuestran una metodología apropiada, diseñada con una asignación al azar y cegamiento apropiado, que permiten analizar la eficacia de las inyecciones intraarticulares de $\mathrm{AH}$ en comparación con 
J. Jara Armijos, B. Hidalgo Andrade, B. Velásquez Ron. Eficacia del ácido hialurónico en el tratamiento de los trastornos temporomandibulares. Revisión sistemática

\begin{tabular}{|c|c|c|c|}
\hline AUTOR/REVISTA/AÑO & $\begin{array}{l}\text { TIPO DE } \\
\text { TRATAMIENTO }\end{array}$ & $\begin{array}{l}\text { PERIODO DE } \\
\text { SEGUIMIENTO }\end{array}$ & $\begin{array}{l}\text { PROTOCOLO DE APLICACIÓN } \\
\text { DE ÁCIDO HIALURÓNICO }\end{array}$ \\
\hline $\begin{array}{l}\text { Fernandez Ferro M. Journal of } \\
\text { Cranio-Maxillo-Facial Surgery. } \\
2017 \text { (22) }\end{array}$ & $\begin{array}{l}\text { Inyección intraarticular } \\
\text { de AH versus Plasma } \\
\text { Rico en Factor de } \\
\text { Crecimiento (PRFC) }\end{array}$ & $\begin{array}{l}3-6-12-18 \\
\text { meses }\end{array}$ & $\begin{array}{l}\text { Mediante artrocentesis se aplicó sobre el disco y } \\
\text { el compartimiento superior } 5 \mathrm{ml} \text { en total de PRFC } \\
\text { obtendio del paciente (Grupo 1) y AH de alto peso } \\
\text { molecular } 1200 \mathrm{Kda} \text { (Grupo 2) }\end{array}$ \\
\hline $\begin{array}{l}\text { Su Naichuan. Journal of } \\
\text { Cranio-Maxillo-Facial Surgery. } \\
2014 \text { (23) }\end{array}$ & $\begin{array}{l}\text { Inyección intraarticular } \\
\text { de AH más clohoridrato } \\
\text { de glucosamina (GS) por } \\
\text { vía oral }\end{array}$ & $\begin{array}{l}\text { Base } \\
1-3-6 \text { meses }\end{array}$ & $\begin{array}{l}5 \text { ciclos de inyecciones intraarticulares sema- } \\
\text { nales }(1 \text { por semana) de } 2 \mathrm{ml} \text { de } A H+0.24 \mathrm{~g} \text { de } \\
\text { cloridrato de GS por vía oral junto con } 0.48 \mathrm{~g} 3 \\
\text { veces al día de GS por tres meses }\end{array}$ \\
\hline $\begin{array}{l}\text { Xao Cen. } \\
\text { Oral Diseases. 2018. (24) }\end{array}$ & $\begin{array}{l}\text { Inyección intraarticular } \\
\text { de AH más GS oral }\end{array}$ & $\begin{array}{l}\text { Base } \\
1 \text { mes - } 1 \text { año }\end{array}$ & $\begin{array}{l}\text { Grupo 1: Inyecciones intraarticulares } \\
\text { de } 1 \mathrm{ml} \text { de AH } 1 \text { vez por semana x } 4 \text { semanas } \\
\text { más } 2 \text { tabletas de GS de } 240 \mathrm{mg} \\
3 \text { veces al día por } 3 \text { meses. } \\
\text { 4Grupo 2: Inyecciones in5traarticulares de } 1 \mathrm{ml} \text { de } \\
\text { AH } 1 \text { vez por semana } 4 \text { semanas más } 2 \text { tabletas } \\
\text { de } 240 \mathrm{mg} \text { de placebo } 3 \text { veces al día por } 3 \text { meses. }\end{array}$ \\
\hline
\end{tabular}

Korkmaz Y, et al . Journal of Oral and Maxillo-Facial Surgery 2016 (25)

\section{Inyección intraarti-} cular de AH versus otras modalidades de terapia

\section{Base}

6 meses

\section{Artrocentesis más Base}

Comert Kilicc.

International Journal of Oral and

Maxillofacial Surgery.2016 (7)

aplicación de AH versus rico en plaquetas (PRP)

Zeliha Gencer

Journal of Cranio-Maxillo-Facial Surgery. 2014 (26)
Inyecciones intraarticulares de AH, tenoxicam (TX) y betametasona (CS) 3-6- 12 meses

Pacientes divididos en 4 grupos. Grupo 1 : Control, Grupo2: 1 sola inyección de AH

$(1 \mathrm{ml})$ en el espacio superior de la ATM. Grupo 3: 2 5inyecciones de AH de $1 \mathrm{ml}$ una a6l inicio del estudio y otra un m7es despues. Grupo 4: Terapia férula oclusal

Grupo 1: Artrocentesis más inyección de $1 \mathrm{ml} \mathrm{PRP} \mathrm{+} \mathrm{inyección} \mathrm{intraarticular}$ mensual de $1 \mathrm{ml}$ de PRP durante 4 meses. Grupo 2: Artrocentesis más inyección de $1 \mathrm{ml}$ de $\mathrm{AH}$ de bajo peso molecular (500-700Kda)

Pacientes se dividieron en 4 grupos: Grupo 1: Inyección intraarticular de AH de $10 \mathrm{mg} / \mathrm{m}$ (2mI). Grupo 2: Inyección intraarticular de betametasona de $7.0 \mathrm{mg} / \mathrm{ml}$ Grupo 3:Inyección intraarticular de Tenoxicam (20 mg/ml) Grupo 4: Inyección intraarticular de solución salina (grupo control).

Ciclo de 5 inyecciones semanales de AH después de artrocentesis para reducir síntomas en pacientes con osteoartritis de ATM

Triantaffilidou K

The Journal of Craneofacial Surgery. 2013 (14)

Oral and Maxillofacial Surgery. $2013(7)$

Inyección intraarticular de $\quad 1,3,6,12$ meses AH sin artrocentesis en pacientes con osteoartritis frente a pacientes que recibieron terapia con AINES por 1 mes

Guarda-Nardini L. Journal of Oral Rehabilitation. 2011 (19)

Morey- Mas M.

Journal of Oral and Maxillofacia Surgery. 2010 (17)

Manfredini D

International Journal of Oral and Maxillofacial Surgery. 2009 (10)
Ciclo de 5 inyecciones (1 por semana) de AH más artrocentesis en paceintes con enfermedad degenerativa de ATM

Comparación de una inyección única intraarticular de AH frente a inyección de lactato de Ringer luego de artroscopia en paceintes con TTM

Artrocentesis más inyecciones intraarticulares cíclicas de $\mathrm{AH}$ en pacientes con osteoartritis de ATM

\section{Base \\ 6 meses \\ 5 artrocentesis (1 por semana) más inyección única de $\mathrm{AH}(1 \mathrm{ml})$}

inyecciones (1 por semana) de $1 \mathrm{ml}$ de $\mathrm{AH}$ de alto peso molecular inyectado en el espacio superior de ATM Grupo B: meloxicam de $7.5 \mathrm{mg} 2$ veces al día por 1 mes.

$\begin{array}{ll}\text { Base } & \text { Artrocentesis más ciclo de } 5 \text { inyecciones ( } 1 \text { por } \\ 1 \text { semana } & \text { semana) de } 1 \mathrm{ml} \mathrm{de} \mathrm{AH} \mathrm{mediante} \mathrm{protocolo} \mathrm{de} \\ 1-3 \text { meses } & \text { una sola aguja }\end{array}$

Base

6 meses

Grupo 1: Artroscopia más 1ml de AH (hialorunato sódico) en el espacio superior de ATM

Grupo 2: Artroscopia más lavado con lactato de ringer

Base 1 semana
La artrocentesis más inyecciones de más artrocentesis mostró mejoCiclo de 5 inyecciones (1 por semana) de Las inyecciones cíclicas de AH meobtenido de hialuronato sódico puro buffe- osteoartitis de ATM especialmente rado en solución salina

\section{RESULTADOS \\ CONSLUIONES}

En términos de dolor

y resultados a largo plazo el

PRFC es mejor frente al AH.

En términos de mejora de la

calidad de vida y reducción de

molestias y dolor la GS por vía ora

potencia el efecto del AH.

La administración de GS oral como 5 (Alto) terapia adyuvante a la inyeccción de AH tiene un gran impacto en el alivio del dolor de los pacientes.

Todos los métodos de tratamiento: $\mathrm{AH} \quad 4$ (Alto) una sola dosis, doble dosis y férula oclusal fueron más efectivos que ningún tratamiento. Y el tratamiento de doble inyección fue más efectivo que la terapia con férula oclusal. PRP no son superior a la artrocentesis más una única inyección de AH.

Los pacientes mostraron mejor alivio del dolor con las inyecciones de $\mathrm{AH}$ comparado con las inyecciones de CS y TX. Los grupos de CS y Tx tuvieron mejores resultados que el grupo control pero el alivio del dolor del TX disminuyo desde la primera hasta la 6 semana de seguimiento.

Los valores en a EVA del dolor decrecieron significativamente con el uso del protocolo de 5 inyecciones más artrocentesis luego del tratamiento y hasta un $61 \%$ más 6 meses después.

La terapia de 5 inyecciones intraar- 3 (Medio) ticulares semanales sin artrocentesis es eficaz en la reducción de dolor y mejora de la apertura buca durante el tratamiento y se mantiene hasta un año mientras que en el grupo de pacientes tratados con AINES solo se obtuvo mejora durante el mes de tratamiento

El protocolo de 5 inyecciones rías respecto al dolor y apertura bucal en el $85 \%$ de los pacientes y se mantuvo hasta los 3 meses.

El lavado intraarticular con AH luego 5 (Alto)

de artroscopia es efectivo en la reducción del dolor en pacientes con TTM con significancia estadistíca a los 14 días del postoperatorio y el efecto se mantiente hasta 6 meses después.

VALOR

ESCALA JADA

3 (Medio)

1 (Bajo)

4 (Alto)

4 (Alto)

5 (Alto)

2 (Bajo)0

3 (Medio) dolor y mejora de apertura bucal,

los cuales se mantienen hasta 6 meses después. 
AVANCES EN ODONTOESTOMATOLOGÍA

Vol. 36 - Núm. 1 - 2020

\section{AUTOR/REVISTA/AÑO TIPO DE TRATAMIENTO}

Tuncel U.

Journal of Craneofacial Surgery.

2012 (12)

Inyecciones repetidas de

AH en ATM para mejorar

síntomas en pacientes

con patología de disco en

fase temprana

Guarda-Nardini L.

Surgery. 2012 (11)

Comparación del efecto

de técnica de una sola

aguja vs 2 agujas en

artrocentesis

Ozdamar Serap. Journal of Oral

Rehabilitacion. 2016 (16)

\section{PERIODO DE PROTOCOLO DE APLICACIÓN SEGUIMIENTO DE ÁCIDO HIALURÓNICO}

Base

$1-6$ meses
Inyección intraarticular de hialuronato sódico $(15 \mathrm{mg} / 1 \mathrm{ml}) 1 \mathrm{ml} 2$ veces a la semana luego de artrocentesis con $200 \mathrm{ml}$ de lactato de Ringer, y luego 3 veces más (inyecciones de AH) con intervalos semanales.
Journal of Oral and Maxillofacial

1-3- 6 meses

Ciclo de 5 inyecciones intrarticulares de AH de bajo peso molecular $(1 \mathrm{ml})$ más artrocentesies con técnica de 2 agujas versus el mismo protocolo con técnica de 1 sola aguja.

\begin{tabular}{|c|c|c|}
\hline $\begin{array}{l}\text { Artrocentesis con Hialuro- } \\
\text { nato de sodio Artrocente- } \\
\text { sis con solución salina. }\end{array}$ & $\begin{array}{l}\text { Preoperatorio } \\
1 \text { semana } \\
1 \text { mes } \\
3 \text { mes } \\
\text { Postoperatorio }\end{array}$ & $\begin{array}{l}\text { En el grupo 1: inyección intraarticular con } 2 \mathrm{ml} \\
\text { de hialuronato sódico (Orthovisc® } 30 \mathrm{mg} / 2 \mathrm{~mL} \\
\text { usando una jeringa estéril. } \\
\text { Grupo } 2 \mathrm{ml} \text { de solución salina 0,9\% con la jering } \\
\text { del fabricante. }\end{array}$ \\
\hline
\end{tabular}

\section{Artrocentesis sola 1 día después}

Artrocentesis y aplicación 1 semana de Hialuronato de Sodio 3 semanas

3 meses

6 meses

Journal of Oral and Maxillofacia surgery. $2016(15)$

\section{Inyección de ácido hia-} lurónico en los espacios articulares superior $e$ inferior
Antes del trata-

miento

3 meses

9 meses
Se colocó $2 \mathrm{ml}$ de lactato de Ringer para distender el espacio superior de la articulación y se insertó una segunda aguja para proporcionar la salida de la solución (grupo 1). Después del procedimiento, en el grupo 2 se inyectó $1 \mathrm{ml}$ de hialuronato sódico en el espacio superior de la articulación.

Ciclo de tres inyecciones, cada dos semanas de $1 \mathrm{ml}$ ácido hialurónico.

Journal of Oral and Maxil
cial Surgery. 2014. (20)

Artrocentesis y ácido hialurónico
Preoperatorio

1 mes

3 meses

6 meses

meses

Artrocentesis con aplicación de ácido hialurónico Artrocentesis sin aplicación de ácido hialurónico

\section{Tolga Korkmaz.} 2016 (28)

Inyección de HA

Doble inyección de HA Terapia de férula - 4 años

\section{6 meses}

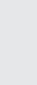

Journal of Oral Science. 2019
(18)
Tolga Korkmaz.
Oral and Maxillofacial Surgery.
2016 (28)

Antes del tratamiento - un año

Małgorzata Pihut.

Folia medica Cracoviensia. 2017 (4) tar - Dos meses
Inyección de ácido hialurónico Inyección de plasma rico en plaquetas
Después de inyec- Se utilizó un $0.4 \mathrm{ml}$ de ácido hialurónico - Se utilizó un $0.4 \mathrm{ml}$ de ácido hialurónico
al $1.5 \%$ para la inyección intraarticular en
un grupo.

Se realizó la artrocentesis, se utilizó 250 $\mathrm{ml}$ de lactato de Ringer para irrigar y $1 \mathrm{ml}$ de ácido hialurónico (ORTHOVISC) para inyectar en el espacio superior.

La artrocentesis se realizó con técnica de dos agujas y con solución de Ringer (grupo A).

En el otro grupo (AS) se combinó la técnica anterior con la inyección intraarticular de aproximadamente $1 \mathrm{~mL}$ de Synvisc (Genzyme Co.).

Se inyectó un volumen de $1 \mathrm{~mL}$ de solución de alto peso molecular. Un mes más tarde, la inyección de HA se repitió para la técnica de inyección de doble HA. Un grupo de este estudio se sometió al uso de una férula estabilizadora por la noche de una férula estabilizad.

En otro grupo se recogió sangre de los pacientes para centrifugar y formar el plasma rico en plaquetas que se usó directamente. Las inyecciones se realizaron 3 veces a intervalos de 2 semanas

Gurung Tikaram. National Journal of Maxillofacia Surgery. 2017 (8)

$\begin{array}{ll}\text { Artrocentesis } & 1 \text { día } \\ \text { Artrocentesis más } & 5 \text { días } \\ \text { inyección intraarticular } & 7 \text { días } \\ \text { de sodio HA } & 4 \text { semanas } \\ & 6 \text { semanas } \\ & 12 \text { semanas. }\end{array}$

En un grupo se realizó artrocentesis con solución de lactato de Ringer en un ciclo de 5 artrocentesis ( 1 por semana).En el otro grupo se efectuó la artrocentesis con solución de lactato de Ringer más inyección intraarticular de sodio $\mathrm{HA}(0,5 \mathrm{ml})$ en el espacio articular superior en un ciclo de 5 artrocentesis (1 por semana).

\section{RESULTADOS/} CONSLUIONES

AH en protocolo cíclico tiene efectos beneficos en signos clínicos y síntomas subjetivos en pacientes con patología de disco articular en fase temprana, especialmente en apertura máxima y disminución del dolor al mes y 6 meses de seguimiento.

A los 6 meses la efectividad de ambos protocolos fue similar con mejoría respecto a los datos base en dolor, eficiencia de masticación y movimientos mandibulares.

Disminución significativa en los niveles de MPO entre en el grupo de ácido hialurónico

Las puntuaciones VAS y MMO disminuyeron en el transcurso del tiempo después de la artrocentesis y no difieren entre HAOSS.

A los 6 meses hubo aumento de la apertura bucal, mejoría en la eficiencia masticatoria y reducción de dolor en los dos grupos, sin embargo la artrocentesis con ácido hialurónico es obtuvo resultados superiores.

La función TMJ mejoró en la mayoría de los pacientes a los 3 meses con movimiento mandibular, alivio de dolor, morfología condilar y el aumento de MMO. La evaluación del CBCT mostró un efecto de mejoría en la osteoartritis después de la inyección inferior. Los cambios radiográficos más comunes fueron: nueva formación ósea y la disolución de osteofitos.

La máxima apertura incisal, excursión y protrusiva mejoraron en comparación al preoperatorio.El dolor disminuyó significativamente. Los pacientes Wilkes III mejoraron más que los pacientes Wilkes II.

La artrocentesis de la ATM redujo el dolor y mejoró la función después de la observación a corto y largo plazo. La inyección de hialuronato sódico no tuvo efecto adicional después de 4 años de seguimiento

Los grupos de inyección de HA presentaron una mejora superior con respecto al dolor, MMO y calidad de vida en comparación con el grupo de la férula de estabilización.

Hubo una disminución de los síntomas individuales, pero los resultados de la regresión del dolor entre dos grupos no difirieron estadísticamente
Se observó una reducción significativa del dolor y mejoría de MMO y de los movimientos en ambos grupos; sin embargo, la artrocentesis con HA de sodio fue superior a la artrocentesis sola.

\section{5(Alto)}

5 (Alto) 1 (Bajo)

3 (Medio)
VALOR ESCALA JADA

3 (Medio)

4 (Alto)

5 (Alto)

4 (Alto)

5 (Alto)

1(Ваjo)

(n)




\section{AUTOR/REVISTA/AÑO TIPO DE TRATAMIENTO \\ Tang Ya-Ling \\ Journal of Oral Surgery, Oral Medicine, Oral Pathology, Oral Radiology, and Endodontology. 2010 (19) \\ nato de sodio \\ Versus inyección de \\ solución salina fisio- \\ lógica}

PERIODO DE PROTOCOLO DE APLICACIÓN SEGUIMIENTO DE ÁCIDO HIALURÓNICO

6 semanas
Las inyecciones se colocaron en el espacio

superior intraarticular en intervalos semanales (5 semanas). Un grupo recibió 5 inyecciones de hialuronato de sodio ( $1 \mathrm{ml})$ con peso molecular de 1500-2500 kDa, mientras que en el otro grupo se aplicaron 5 inyecciones de solución salina fisiológica. Se recogió fluido sinovial antes y después del tratamiento $(1$ semana después de la última inyección).

\section{RESULTADOS/} CONSLUIONES

El rol del ácido hialurónico en regular el sistema plasminógeno provee una nueva visión en relación al mejoramiento de la sintomatología de la osteoartritis en la articulación temporomandibular
VALOR ESCALA JADA

3 (Medio)

\begin{tabular}{|c|c|c|c|c|c|c|c|c|c|}
\hline & $\begin{array}{c}\text { Random } \\
\text { sequence } \\
\text { generation } \\
\text { (selection bias) }\end{array}$ & $\begin{array}{c}\text { Allocation } \\
\text { concealment } \\
\text { (selection bias) }\end{array}$ & \begin{tabular}{|c|} 
Blinding of \\
participants and \\
personelle(per- \\
formance bias)
\end{tabular} & $\begin{array}{l}\text { Blinding of } \\
\text { outcome } \\
\text { assesment } \\
\text { (detection bias) } \\
\text { (patient-repor- } \\
\text { ted outcomes }\end{array}$ & \begin{tabular}{|c} 
Blinding of \\
outcome asses- \\
ment (detection \\
bias) (all-cause \\
mortality)
\end{tabular} & $\begin{array}{c}\text { Incomplete } \\
\text { outcome da- } \\
\text { ta(attrition bias) } \\
\text { (short-term [2-6 } \\
\quad \text { weeks] }\end{array}$ & $\begin{array}{c}\text { Incomplete } \\
\text { outcome da- } \\
\text { ta(attrition bias) } \\
\text { (short-term [2-6 } \\
\text { weeks] }\end{array}$ & $\begin{array}{c}\text { Incomplete } \\
\text { outcome da- } \\
\text { ta(attrition bias) } \\
\text { (long-term [>6 } \\
\text { weeks] }\end{array}$ & $\begin{array}{c}\text { Selective repor- } \\
\text { ting (reporting } \\
\text { bias) }\end{array}$ \\
\hline Fernandez-Ferrero, M et al 2017 & $\oplus$ & ( & - & 0 & ? & ( & + & + & + \\
\hline Su, N et al 2014 & - & ( & ?? & ? & $?$ & $\oplus$ & + & ( & $\oplus$ \\
\hline Cen, $\mathrm{X}$ et al 2018 & $\oplus$ & $\oplus$ & $\oplus$ & $\oplus$ & $\oplus$ & $\oplus$ & $\oplus$ & $\oplus$ & + \\
\hline Korkmaz, Y et al 2016 & + & + & + & - & $?$ & + & + & ( & + \\
\hline Comert Kilicc, S et al 2016 & $\oplus$ & † & $\oplus$ & - & $?$ & $\oplus$ & $\oplus$ & $\oplus$ & + \\
\hline Gencer, Z et al 2014 & + & + & + & + & + & + & + & + & + \\
\hline Manfredini D. et al 2013 & - & ( & ( & $?$ & - & + & + & + & $\oplus$ \\
\hline Triantaffilidou, K et al 2013 & $?$ & $\oplus$ & $\oplus$ & $\oplus$ & $\oplus$ & $\oplus$ & † & $\oplus$ & + \\
\hline Guarda-Nardini, L et al 2011 & $?$ & ( & + & + & + & + & + & + & + \\
\hline Morey- Mas, M. et al 2010 & + & + & + & + & + & + & + & + & $\oplus$ \\
\hline Manfredini, D et al 2009 & - & ( & ( & † & ( & ( & † & $\oplus$ & + \\
\hline Tuncel U. 2012 & - & ( & ( & + & + & + & + & + & 0 \\
\hline Guarda-Nardini L. 2012 & + & ( & + & + & ( & $\oplus$ & ( & + & - \\
\hline Bergstrand S, et al 2019 & $\oplus$ & ( & $\oplus$ & $\oplus$ & + & + & $\oplus$ & + & 0 \\
\hline Tolga Korkmaz Y. et al 2016 & $\oplus$ & $\oplus$ & $\oplus$ & $\oplus$ & $\oplus$ & $\oplus$ & $\oplus$ & $\oplus$ & + \\
\hline Małgorzata P. et al 2017 & 0 & + & $\oplus$ & $\oplus$ & 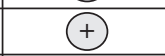 & $\oplus$ & $\oplus$ & + & 0 \\
\hline Gurung T. et al 2017 & $\oplus$ & 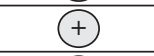 & $\oplus$ & + & + & 0 & - & + & + \\
\hline Tang Ya-Ling, et al 2010 & $\oplus$ & $\oplus$ & + & $\oplus$ & (†) & - & 0 & $\oplus$ & $\oplus$ \\
\hline
\end{tabular}

otras pruebas intraarticulares y protocolos.

Dentro de los tratamientos iniciales invasivos para el tratamiento de TTM se incluye la artrocentesis, que consiste en lavado articular con solución de lactato de Ringer o solución salina para la eliminación de componentes inflamatorios. Varios estudios han evaluado la eficacia de la artrocentesis asociada o no con inyecciones de $\mathrm{AH}$ en la reducción de la intensidad del dolor y la mejora función de la apertura bucal en pacientes con TTM, sin embargo en esta revisión todos los autores encontraron que los tratamientos combinados con $\mathrm{AH}$ obtuvieron resultados superiores.

El plasma rico en factor de crecimiento (PRFC) luego de artrocentesis también se ha comparado frente al $\mathrm{AH}$ obteniendo mejores resultados para el PRFC en términos de dolor, resultados de apertura bucal a largo plazo, en primer lugar, el PRFC ayuda a restaurar los niveles de HA endógeno, componente principal en la disminución del líquido sinovial en los procesos degenerativos de ATM, al estimula su liberación proporcionando el beneficio mecánico, antiinflamatorio y analgésico que esto implica; además PRFC y $\mathrm{AH}$ comparten las mismas vías metabólicas de acción involucradas en la activación y regulación de algunos procesos fisiológicos, especialmente en la relación con la actividad de la interleucina-1, que experimentalmente mejora las propiedades biológicas del $\mathrm{AH}^{(1)}$. Estos 2 mecanismos descritos el PRFC y AH se superponen complementándose entre sí e incluso se podría pensar en un posible efecto sinérgico entre las dos sustancias. 
El plasma rico en plaquetas y $\mathrm{AH}$ demuestra propiedades curativas, diversos estudios tratan de compararlos con el fin de establecer cuál es la mejor elección. Cömert Kiliç, et al en su investigación demostró que los dos tratamientos proveen mejoras significativas en cuanto a EVA(escala visual análoga) y la disminución del dolor a la máxima apertura interincisal $(\mathrm{MIO})^{(7)}$, la artrocentesis con PRT no es superior a la artrocentesis con $\mathrm{AH}$, este último resulta ser más aceptado por los pacientes. ${ }^{(4)}$ contrasta los dos tratamientos sin previa artrocentesis con resultados control de síntomas, la regresión del dolor no difirió significativamente entre los dos grupos.

El protocolo de aplicación cíclica usado por su eficacia en otras articulaciones, especialmente rodilla, es una nueva propuesta en tratamiento con AH en los TTM. ${ }^{\left({ }^{8}\right)}$ en su estudio cíclico de cinco artrocentesis semanales, corrobora que la artrocentesis combinada con la inyección de $\mathrm{AH}$ funciona mejor que la artrocentesis sola; iguales datos obtienen Guarda-Nardini, L, et al. ${ }^{(9)}$, Manfredini, D, et al. ${ }^{(10)}$, Guarda-Nardini, L. et al. ${ }^{(11)}$ y Tuncel U. (12) quienes confirman la mejoría de síntomas especialmente dolor, apertura bucal en los pacientes con patología articular temprana y degenerativa que se mantienten incluso hasta 6 meses después.

No se detalla en la mayoría de los estudios el peso molecular del AH aunque varios autores concuerdan que alto peso molecular da como resultado una mayor viscosidad y una menor facilidad de difusión del $\mathrm{AH}$, por lo que se maneja $\mathrm{AH}$ con pesos moleculares medios o bajos en la mayoría de los estudios.

Guarda-Nardini et al. utilizaron el protocolo de aplicaciones cíclicas de cinco sesiones de artrocentesis asociadas con la suplementación de $\mathrm{AH}$ de peso molecular medio o bajo encontrando que el protocolo fue superior incluso después de 6 meses de seguimiento. ${ }^{(9-11)}$

Manfredini et al. compararon diferentes protocolos para el tratamiento de la osteoartritis que incluyeron artrocentesis mas suplementación única y cíclica de $\mathrm{AH}$ de peso molecular medio obteniendo en ambos estudios reducción significativa del dolor y mejora de la apertura bucal luego del tratamiento que se mantuvieron hasta 6 meses después. ${ }^{(10-13)}$
Triantaffilidou, et al. ${ }^{(14)}$ aplicaron de forma cíclica el AH con alto peso molecular sin artrocentesis y demostró que esta terapia es capaz de aliviar la intensidad del dolor y aumento la apertura bucal en pacientes con osteoartrosis de la ATM durante el tratamiento, manteniéndose hasta el año de seguimiento; en comparación con pacientes que recibieron medicación con AINES los cuales obtuvieron el alivio únicamente por el período de duración de la terapia y 1 mes después de finalizar el tratamiento.

Patel et al. ${ }^{(15)}$ también compara la artrocentesis sola y artrocentesis combinada con ácido hialurónico donde a los 6 meses se obtuvo aumento de la apertura bucal, más eficiencia masticatoria y reducción de dolor en los dos grupos, la artrocentesis con ácido hialurónico no dejo de tener resultados superiores. Ozdamar S. et al. ${ }^{(16)} \mathrm{com}-$ paró la artrocentesis con AH y artrocentesis con solución salina obteniendo que el dolor en el EVA y máxima apertura bucal mejoraron luego de la artrocentesis con la aplicación de $\mathrm{AH}$ y solución salina; similares resultados reportan Morey-Mas et al. ${ }^{(17)}$ que compara artrocentesis con inyección única de $\mathrm{AH}$ frente a lactacto de ringer, obteniendo resultados significativamente superiores a los 14 días posoperatorios que se mantuvieron hasta 6 meses después en el grupo de $\mathrm{AH}$.

En cambio, investigaciones como la de Bergstrand et al. ${ }^{(18)}$ indican que la artrocentesis por si sola reduce el dolor, mejora la función a corto y largo plazo, el $\mathrm{AH}$ no tiene un efecto adicional después de 4 años seguimiento.

El diagnóstico por imágenes de alteraciones de la ATM, como la resonancia magnética (RM) y la tomografía computarizada (TC) fueron los métodos más utilizados para los exámenes de tejidos blandos y duros, respectivamente. Estudios recientes sugieren que el sistema activado de plasminógeno podría estar implicado en la reabsorción del cartílago y el hueso en los trastornos temporomandibulares $^{(19)}$, la evaluación de los beneficios del AH a través de imágenes es escasa; sin embargo Li et al. ${ }^{(20)}$ analizaron los resultados de la aplicación de $\mathrm{AH}$ en los espacios intrarticulares superior e inferior a través de TC de haz cónico en pacientes con osteoartritis observando que luego de la inyección se logró remodelación condilar, a su vez se logró un mejor funcionamiento mandibular con resultados de imagen que lo confirman y la nueva 
formación de tejido óseo y disolución de osteofitos a los 3 meses del tratamiento.

Dentro de los TTM descritos, la osteoartritis fue la enfermedad más prevalente en todos los ensayos clínicos incluidos, los pacientes con este trastorno tienden a mostrar una reducción en la concentración de $\mathrm{AH}$ intraarticular debido a la despolimerización por especies reactivas de oxígeno y la producción de moléculas de ácido con un peso molecular más bajo de lo normal, lo que produce una reducción en la lubricación, aumento en el estrés mecánico de las articulaciones, resultando en la progresión clínica y radiográfica de la enfermedad ${ }^{(21)}$.

\section{CONCLUSIONES}

Los estudios incluidos en esta revisión sistemática presentaron diferencias en la ejecución de los protocolos de aplicación, ciclos, dosis, y peso molecular del ácido hialurónico, por lo que no se puede establecer un protocolo "Gold estándar" para pacientes con disfunción temporomandibular, sin embargo se pueden extraer las siguientes conclusiones:

-El ácido hialurónico mejora en general la mayoría de los síntomas de los TTM como son: dolor, apertura bucal limitada, movimientos mandibulares y calidad de vida de los pacientes.

- La combinación de AH con técnicas como artroscopia o artrocentesis, potencializa los efectos a corto y largo plazo.

- La aplicación cíclica de inyecciones de AH en diferentes protocolos demostró una mejoría a largo plazo de los síntomas de los diferentes TTM que se extendieron hasta 12 meses luego de su aplicación.

\section{Conflicto de Interés.}

No existe conflicto de intereses de los autores

\section{BIBLIOGRAFIA}

1. Fernández-Ferro $M$, Fernández-Sanromán J, Blanco-Carrión A, Costas-López A, López-Betancourt A, Arenaz-Bua J, et al. Comparison of intra-articular injection of plasma rich in growth factors versus hyaluronic acid following arthroscopy in the treatment of temporomandibular dysfunction: A randomised prospective study. J Cranio-Maxillofacial Surg [Internet]. 2017 Apr;45(4):449-54. Available from: https://linkinghub.elsevier.com/retrieve/pii/S1010518217300215

2. Jung YW, Park SH, On SW, Song SI: Correlation between clinical symptoms and magnetic resonance imaging findings in patients with temporomandibular joint internal derangement. J Korean Assoc Oral Maxillofac Surg 41(3): 125e132, 2015. Doi: 10.5125/ jkaoms.2015.41.3.125.

3. Patel P, Idrees F, Newaskar V, Agrawal D. Sodium hyaluronate: an effective adjunct in temporomandibular joint arthrocentesis. Oral Maxillofac Surg. 2016; 24(4): p. 3-8. Avaliable from https://link.springer.com/article/10.1007\%2Fs10006-016-0581-2

4. Malgorzata P, Malgorzata G, Ceranowicz P. Evaluation of remission of temporomandibular joints pain as a result of treatment of dysfunction using intraarticular injection. Folia medica Cracoviensia. 2017; 57(3): p. 57-65. Avaliable from https://www.ncbi.nlm.nih.gov/ pubmed/29263455

5. Hegab AF, Ali HE, Elmasry M, Khallaf MG: Platelet-rich plasma injection as an effective treatment for temporomandibular joint osteoarthritis. J Oral Maxillofac Surg 73(9): 1706e1713, 2015

6. Guidolin D. Intra-articular 500-730 kDa hyaluronan (Hyalgan $®)$ therapy in the management of osteoarthritis. Can a specific therapeutic profile be defined? Eur Rev Med Pharmacol Sci [Internet]. 2018;22(14):4698-719. Available from: http://www.ncbi.nlm.nih.gov/pubmed/30058706

7. Cömert Kiliç $S$, Güngörmü囚 $M$. Is arthrocentesis plus platelet-rich plasma superior to arthrocentesis plus hyaluronic acid for the treatment of temporomandibular joint osteoarthritis: a randomized clinical trial. Int $\mathrm{J}$ Oral Maxillofac Surg [Internet]. 2016 Dec;45(12):1538-44. Available from: https://doi.org/10.1016/j. ijom.2016.06.009 
8. Gurung T, Singh R, Mohammad, Pal, Mahdi AA, Kumar. Efficacy of arthrocentesis versus arthrocentesis with sodium hyaluronic acid in temporomandibular joint osteoarthritis: A comparis. National of Journal of maxillofacial Surgery. 2017; 8(1): p. 41-49. Avaliable from https://www.ncbi.nlm.nih.gov/pubmed/28761275

9. Guarda-Nardini L, Ferronato G, Favero L, Manfredini D. Predictive factors of hyaluronic acid injections short-term effectiveness for TMJ degenerative joint disease. J Oral Rehabil [Internet]. 2011 May;38(5):315-20. Available from: http://doi.wiley.com/10.1111/j.13652842.2010.02164.x

10. Manfredini D, Bonnini S, Arboretti R, Guarda-Nardini L. Temporomandibular joint osteoarthritis: an open label trial of 76 patients treated with arthrocentesis plus hyaluronic acid injections. Int J Oral Maxillofac Surg [Internet]. 2009 Aug;38(8):827-34. Available from: https://linkinghub.elsevier.com/retrieve/pii/S090150270900839X

11. Guarda-Nardini L, Ferronato G, Manfredini D. Two-needle vs. single-needle technique for TMJ arthrocentesis plus hyaluronic acid injections: a comparative trial over a six-month follow up. Int $\mathrm{J}$ Oral Maxillofac Surg [Internet]. 2012 Apr;41(4):506-13. Available from: https://linkinghub.elsevier.com/retrieve/pii/ S0901502711014937

12. Tuncel $U$. Repeated sodium hyaluronate injections following multiple arthrocenteses in the treatment of early stage reducing disc displacement of the temporomandibular joint: A preliminary report. Journal of Cranio-Maxillo-Facial Surgery 40 (2012) 685 e689. doi:10.1016/j.jcms.2011.12.003

13. Manfredini D, Favero L, Del Giudice A, Masiero S, Stellini E, Guarda-Nardini L. Axis II psychosocial findings predict effectiveness of TMJ hyaluronic acid injections. Int J Oral Maxillofac Surg [Internet]. 2013 Mar;42(3):364-8. Available from: http://dx.doi.org/10.1016/j. ijom.2012.10.033

14. Triantaffilidou K, Venetis G, Bika O. Efficacy of Hyaluronic Acid Injections in Patients
With Osteoarthritis of the Temporomandibular Joint. A Comparative Study. J Craniofac Surg [Internet]. 2013 Nov;24(6):2006-9. Available from: http://content.wkhealth.com/ linkback/openurl?sid=WKPTLP:landingpageEan=00001665-201311000-00029

15. Patel1 P. Idrees F, Newaskar V, Agrawal D. Sodium hyaluronate: an effective adjunct in temporomandibular joint arthrocentesis. Oral Maxillofac Surg (2016) 20:405-410. DOI 10.1007/s10006-016-0581-2.

16. Ozdamar S, Alev B, Yarat A. The impact of arthrocentesis with and without hyaluronic acid injection in the prognosis and synovial fluid myeloperoxidase levels of patients with painful symptomatic internal derangement of temporomandibular joint: a randomized controlled clinical trial. J. Oral Rehabilitation. 2016; 44(2): p. 1-14. Available from: https://www. ncbi.nlm.nih.gov/pubmed/27973684

17. Morey-Mas MA, Caubet-Biayna J, Varela-Sende L, Iriarte-Ortabe JI. Sodium Hyaluronate Improves Outcomes After Arthroscopic Lysis and Lavage in Patients With Wilkes Stage III and IV Disease. J Oral Maxillofac Surg [Internet]. 2010;68(5):1069-74. Available from: http:// dx.doi.org/10.1016/j.joms.2009.09.039 http://doi.wiley.com/10.1111/j.13652842.2007.01759.x

18. Bergstrand S, Ingstad H, Møystad A, Bjørnland $\mathrm{T}$ T. Long-term effectiveness of arthrocentesis with and without hyaluronic acid injection for treatment of temporomandibular joint osteoarthritis. J Oral Sci. 2019; 61(1): p. 82-88. Avaliable from https://www.jstage.jst.go.jp/ article/josnusd/61/1/61_17-0423/_article

19. Tang YL, Zhu Gq, Hu L, Min Z, Zhang Jy, Shi $\mathrm{Zd}$, et al. Effect of intra- articular administration of sodium hyaluronate on plasminogen activator system in temporomandibular joint with osteoarthritis. Oral Surgery, Oral Medicine, Oral Pathology, Oral Radiology and endodontology. 2010; 109(4): p. 541-547. Avaliable from https://www-clinicalkey-es. bibliotecavirtual.udla.edu.ec/\#!/content/journal/1-s2.0-S1079210409008427

20. Li C, Long X, Deng M, Li J, Hengxing C, Meng. 
J. Jara Armijos, B. Hidalgo Andrade, B. Velásquez Ron. Eficacia del ácido hialurónico en el tratamiento de los trastornos temporomandibulares. Revisión sistemática

Osteoarthritic changes after superior and inferior joint space injection of hyaluronic acid for the treatment of TMJ osteoarthritis with anterior disc displacement without reduction: A Cone-Beam Computed Tomographic Evaluation. Journal of Oral and Maxillofacial Surgery. 2014; 73(2): p. 232-244. Avaliable from https://www.joms.org/article/S02782391(14)01406-2/fulltext

21. Machado E, Machado P, Wandscher VF, Marchionatti AME, Zanatta FB, Kaizer OB. A systematic review of different substance injection and dry needling for treatment of temporomandibular myofascial pain. Int J Oral Maxillofac Surg [Internet]. 2018 Nov;47(11):142032. Available from: https://linkinghub.elsevier. com/retrieve/pii/S0901502718301759

22. Fernandez-Ferro M, Fernandez-Sanroman, J , Blanco-Carrion A, Costas-Lopez A, Lopez-Betancourt A, Arenaz-Bua J, Stavaru Marinescu, B. Comparison of intra-articular injection of plasma rich in growth factors versus hyaluronic acid following arthroscopy in the treatment of temporomandibular dysfunction: A randomised prospective study . Journal of Cranio-Maxillo-Facial Surgery 45 (2017) 449e454. http://dx.doi.org/10.1016/j. jcms.2017.01.010

23. Su N, Yang X, Liu Y, Huang Y, Shi Z. Evaluation of arthrocentesis with hyaluronic acid injection plus oral glucosamine hydrochloride for temporomandibular joint osteoarthritis in oral-health-related quality of life. J Cranio-Maxillofacial Surg [Internet]. 2014 Sep;42(6):846-51. Available from: http://dx. doi.org/10.1016/j.jcms.2013.12.003

24. Cen X, Liu Y, Wang S, Yang X, Shi Z, Liang $X$. Glucosamine oral administration as an adjunct to hyaluronic acid injection in treating temporomandibular joint osteoarthritis. Oral Dis [Internet]. 2018 Apr;24(3):404-11. Available from: http://doi.wiley.com/10.1111/ odi. 12760

25. Korkmaz YT, Altıntas NY, Korkmaz FM, Candırlı C, Coskun U, Durmuslar MC. Is Hyaluronic Acid Injection Effective for the Treatment of Temporomandibular Joint Disc Displacement With Reduction? J Oral Maxillofac
Surg [Internet]. 2016 Sep;74(9):1728-40. Available from: http://dx.doi.org/10.1016/j. joms.2016.03.005

26. Gencer ZK, Özkiri冈 M, Okur A, Korkmaz M, Saydam L. A comparative study on the impact of intra-articular injections of hyaluronic acid, tenoxicam and betametazon on the relief of temporomandibular joint disorder complaints. J Cranio-Maxillofacial Surg [Internet]. 2014 Oct;42(7):1117-21. Available from: https://linkinghub.elsevier.com/retrieve/pii/ S1010518214000432

27. Ungor C, Atasoy K, Taskesen Ã, Pipir C, Yilmaz O. Long-Term Outcome of Arthrocentesis Plus Hyaluronic Acid Injection in Patients With Wilkes Stage II and III Temporomandibular Joint Internal Derangement.. J Craniofac Surg. 2015; 26(7): p. 2104-8. Avaliable from https://europepmc.org/abstract/ med/26468791

28. Tolga Korkmaz , Yılmaz Altıntas , Mehmet Korkmaz F, Candirli C, Coskun U, Cenk Durmuslar $M$. Is hyaluronic acid injection effective for the treatment of temporomandibular joint disc displacement with reduction? Journal of Oral and Maxillofacial Surgery. 2016; 74(9): p. 1728-1740. Avaliable from https://www-clinicalkey-es.ezproxy. javeriana.edu.co/\#!/content/playContent/1-s2.0-S0278239116003396?returnurl=nullEreferrer=null

29. Moldez M, Camones V, Ramos G, Padilla M, Enciso R. Effectiveness of Intra-Articular Injections of Sodium Hyaluronate or Corticosteroids for Intracapsular Temporomandibular Disorders: A Systematic Review and Meta-Analysis. J Oral Facial Pain Headache [Internet]. 2018 Jan;32(1):53-66. Available from: http://quintpub.com/journals/ofph/abstract.php?iss2_id=1502Earticle_id=17975

30. Puissant E, Boonen M. Monocytes/Macrophages Upregulate the Hyaluronidase HYAL1 and Adapt Its Subcellular Trafficking to Promote Extracellular Residency upon Differentiation into Osteoclasts. Tuukkanen J, editor. PLoS One [Internet]. 2016 Oct 18;11(10):e0165004. Available from: http://dx.plos.org/10.1371/ journal.pone.0165004 
31. Talaat WM, Haider M, Kawas S Al, Kandil NG, Harding DRK. Chitosan-Based Thermosensitive Hydrogel for Controlled Drug Delivery to the Temporomandibular Joint. J Craniofac Surg [Internet]. 2016 May;27(3):735-40. Available from: http://insights.ovid.com/ crossref?an $=00001665-201605000-00042$

32. Castaño-Joaqui OG, Muñoz-Guerra MF, Campo J, Martínez-Bernardini G, Cano J. Estado actual de la viscosuplementación con ácido hialurónico en el tratamiento de los trastornos temporomandibulares: revisión sistemática. Rev Española Cirugía Oral y Maxilofac [Internet]. 2017 Oct;39(4):213-20. Available from: https://linkinghub.elsevier.com/retrieve/pii/ S1130055816300508

33. Machado E, Bonotto D, Cunali PA. Intra-articular injections with corticosteroids and sodium hyaluronate for treating temporomandibular joint disorders: a systematic review. Dental Press J Orthod [Internet]. 2013 Oct;18(5):128-33. Available from: http:// www.scielo.br/scielo.php?script=sci_arttextEpid=S2176-94512013000500021 Eln$\mathrm{g}=\mathrm{en} \boldsymbol{t} \operatorname{lng}=\mathrm{en}$

34. Pasquali Ronchetti I, Guerra D, Taparelli F, Boraldi F, Bergamini G, Mori G, et al. Morphological analysis of knee synovial membrane biopsies from a randomized controlled clinical study comparing the effects of sodium hyaluronate (Hyalgan $囚)$ and methylprednisolone acetate (Depomedrol ${ }^{\circledR}$ ) in osteoarthritis. Rheumatology [Internet]. $2001 \mathrm{Feb} ; 40(2): 158-69$. Available from: https://academic.oup.com/ rheumatology/article-lookup/doi/10.1093/ rheumatology/40.2.15

35. Altman RD. Status of hyaluronan supplementation therapy in osteoarthritis. Curr Rheumatol Rep [Internet]. 2003 Jan;5(1):7-14. Available from: http://link.springer.com/10.1007/ s11926-003-0077-6

36. Duygu G, Güler N, Çam B, Kürkçü $M$. The effects of high molecular weight hyaluronic acid (Hylan G-F 20) on experimentally induced temporomandibular joint osteoartrosis: part II. Int J Oral Maxillofac Surg [Internet]. 2011 Dec;40(12):1406-13. Available from: https://linkinghub.elsevier.com/retrieve/pii/

\section{S0901502711012148}

37. Brown T, Laurent U, Fraser. Turnover of hyaluronan in synovial joints: elimination of labelled hyaluronan from the knee joint of the rabbit. Exp Physiol [Internet]. 1991 Jan 1;76(1):125-34. Available from: http://doi.wiley.com/10.1113/expphysiol.1991.sp003474

38. GUARDA-NARDINI L, MASIERO S, MARIONI G. Conservative treatment of temporomandibular joint osteoarthrosis: intra-articular injection of sodium hyaluronate. J Oral Rehabil [Internet]. 2005 Oct;32(10):729-34. Available from: http://doi.wiley.com/10.1111/j.13652842.2005.01505.x

39. Alpaslan C, Bilgihan A, Alpaslan GH, Güner B, Yis MÖ, Erba囚 D. Effect of arthrocentesis and sodium hyaluronate injection on nitrite, nitrate, and thiobarbituric acid-reactive substance levels in the synovial fluid. Oral Surgery, Oral Med Oral Pathol Oral Radiol Endodontology [Internet]. 2000 Jun;89(6):686-90. Available from: https://linkinghub.elsevier.com/retrieve/pii/S1079210400568115

40. Hepguler S, Akkoc YS, Pehlivan M, Ozturk C, Celebi G, Saracoglu A, et al. The efficacy of intra-articular sodium hyaluronate in patients with reducing displaced disc of the temporomandibular joint. $\mathrm{J}$ Oral Rehabil [Internet]. 2002 Jan;29(1):80-6. Available from: http://doi.wiley.com/10.1046/j.13652842.2002.00807.x

41. Grossmann, Januzzi , Iwaki Filho. The use of sodium hyaluronate in the treatment of temporomandibular joint disorders. Rev Dor. São Paulo. 2013; 14(4): p. 301-6. Obtenido de http://www.scielo.br/scielo.php?pi$\mathrm{d}=\mathrm{S} 180600132013000400013 E$ script $=$ sci arttextEtlng=en

42. Iturriaga V, Vásquez B, Manterola C, Del Sol M. Role of Hyaluronic Acid in the Homeostasis and Therapeutics of Temporomandibular Joint Osteoarthritis. Int. J. Morphol. 2017; 35(3): p. 870-876. Obtenido de https://scielo. conicyt.cl/scielo.php?script=sci_abstractEpi$\mathrm{d}=$ S0717-95022017000300012Elng=enEnr$\mathrm{m}=\mathrm{iso}$ 
43. Túrcio KHL, dos Santos DM. Are intra- articular injections of hyaluronic acid effective for treatment of temporomandibular disorders? Systematic review. International Journal Oral and Maxillofacial Surgery. 2016;(45): p. 1531 1537. Obtenido de https://www.ncbi.nlm.nih. gov/pubmed/27374020

44. Iturriaga V, Bornhardt T, Manterola C, Brebi P. Effect of hyaluronic acid on the regulation of inflammatory mediators in ostearthritis of the temporomandibular joint: a systematic review. International Journal of Oral and Maxillofacial Surgery. 2017;(46): p. 590-595. Obtenido de https://www.ncbi.nlm.nih.gov/pubmed/28228347

45. Batifol D, Huart A, Finiels PJ, Nagot N, Jammet P. Effect of intra-articular botulinum toxic injections on temporo-mandibular joint pain. Journal Stomatology Oral \& Maxillofacial Surgery. 2018;(195): p. 1-6.

46. Azevedo Lemos G, Rissi R, Rosa Pimentel E, Palomari ET. Effects of high molecular weight hyaluronic acid on induced arthritis of the temporomandibular joint in rats. Acta Histochemica. 2015; p. 1-10. Obtenido de https://www. semanticscholar.org/paper/Effects-of-high-molecular-weight-hyaluronic-acid-on-Lemos-Rissi/aa5eb03f0030c1 cbdf14d701acf501f02e304145

47. Roshini Srinivas, James, Muthusekhar MR. Efficacy of Hyaluronic Acid in the Treatment of Internal Derangement - Clinical Study. Research Journal Pharmacy and Technology. 2018; 11(8): p. 3483-3485. Obtenido de https://www.researchgate.net/publication/328634122_Efficacy_of_Hyaluronic_ Acid_in_the_Treatment_of_Internal_Deran-
gement_-_Clinical_Study

48. Ferreira, Masterson, Lopes de Lima, de Souza Moura , Oliveira AT, da Silva Fidalgo TK, et al. Efficacy of viscosupplementation with hyaluronic acid in temporomandibular disorders: A systematic review. Journal of Cranio-Maxillo-Facial Surgery. 2018; 46(11): p. 1943-1952. Obtenido de https://www.ncbi. nlm.nih.gov/pubmed/30249483

49. Shinohara T, Izawa $\mathrm{T}$, Mino-Oka $\mathrm{A}$, Mori $\mathrm{H}$, Iwasa A, Inubushi T, et al. Hyaluronan metabolism in overloaded temporomandibular joint. Journal of Oral Rehabilitation. 2016; 43(12): p. 921-928. Obtenido de https://onlinelibrary. wiley.com/doi/abs/10.1111/joor.12443

50. Liu Y, Fei W, Cen X, Xiong Y, Wu J, Wang S, et al. Is there a difference in Intra Articular injections of corticosteroids, Hyaluronate, or Placebo for Temporomandibular osteoarthritis. American Association of Oral and Maxillofacial Surgeons. 2018; 76(3): p. 504-514. Obtenido de https://www.ncbi.nlm.nih.gov/ pubmed/29182905

\section{AUTOR DE CORRESPONDENCIA:}

Jorge Israel Jara Armijos

Departamento de Prótesis, Facultad de Odontología, Universidad de las Américas UDLA, Colon y 6 de diciembre, +59302391000 ext. 3060 . +593980073826. 170523. Quito - Ecuador jorge.jara@udla.edu.ec / jjorge_israel@hotmail.com ORCID ID: https://orcid.org/0000-0002-2395-1349

\section{FUENTE DE FINANCIAMIENTO}

Auspicio de la Universidad de las Américas UDLA (Quito-Ecuador) 